\title{
Physiopathologie du paludisme
}

Les mécanismes des manifestations pathologiques du paludisme sont nombreux et complexes; ils impliquent, entre autres, des altérations érythrocytaires et des perturbations humorales, notamment immunologiques.

\section{Eduardo Dei-Cas \\ Maître de conférences-praticien hospitalier au Chu de Lille}

Pierre Maurois

Chargé de recherches à l'Inserm

Alain Vernes

Professeur à la faculté de médecine de Lille, Directeur de l'Unité 42 de l'Inserm.

\section{ADRESSE}

E. Dei-Cas, P. Maurois, A. Vernes : Inserm $\mathrm{U}_{42}, 369$ rue J. Guesde, 59650 Villeneuved'Ascq. e paludisme constitue aujourd'hui la plus importante endémie parasitaire. Il atteint huit cent millions de personnes dans le monde, étant responsable de un million cinq cent mille morts par an. Cette parasitose est déterminée par des protozoaires sporozoaires intracellulaires du genre Plasmodium. Quatre espèces parasitent l'homme : $P$. vivax, $P$. ovale, $P$. malariae et $P$. falciparum. Cette dernière espèce est habituellement responsable des formes cliniques les plus sévères.

\section{Le cycle parasitaire}

L'homme contracte le paludisme lors de la piqûre de moustiques infectés du genre Anopheles ( figure I). Ceux-ci inoculent des milliers de sporozoïtes*, cellules minces et allongées, mononucléées, capables de pénétrer dans les hépatocytes et de s'y transformer en une masse cytoplasmique plurinucléée ou plasmodiale : le schizonte* hépatique. Celui-ci produit par division asexuée (schizogonie*) 2000 à plusieurs dizaines de milliers (40000 chez $P$. falciparum) [I] de petites cellules uninucléées (mérozoïtes*). Celles-ci, déversées dans la circulation, sont capables de pénétrer dans les érythrocytes où de nouveaux cycles schizogoniques (schizogonie érythrocytaire) vont se succéder périodiquement en donnant lieu aux accès palustres. Lors du développement érythrocytaire, sont aussi générés les gamétocytes*, cellules sexuées capables d'évoluer chez le moustique vers la formation d'un zygote (ookinète). Celui-ci devient un oocyste, à l'intérieur duquel se développeront les sporozoïtes infestants.

L'homme peut aussi contracter le paludisme par voie transfusionnelle, transplacentaire, ou par l'intermédiaire de piqûres effectuées avec des aiguilles souillées par le sang de malades impaludés.

$P$. vivax, $P$. cynomolgi, une espèce infectant les primates et $P$. ovale, peuvent donner de vraies rechutes* (accès palustres survenant des mois ou des années après disparition des premières crises) actuellement expliquées par l'existence d'hypnozoïtes* [I]. P. falciparum, incapable de provoquer de vraies rechutes, peut donner des accès de recrudescence, 
après un intervalle de huit à douze semaines, accès qui seraient dus à la persistance de formes intraérythrocytaires.

\section{Aspects \\ clinico-pathologiques}

Bien que les manisfestations cliniques habituelles du paludisme répondent à une chronobiologie relativement précise, liée au rythme de la schizogonie érythrocytaire, il existe aussi une pathologie palustre moins bien connue, qui s'exprime à long terme et sur laquelle nous reviendrons brièvement à la fin de cet article.

Le phénomène central du paludisme est l'accès qui correspond à la libération, simultanée et massive, de nouvelles formes mérogoniques, associée à la destruction des hématies hôtes qui hébergeaient les schizontes. La périodicité des schizogonies et donc des accès, permet la distinction entre les fièvres tierces (un accès toutes les 48 heures : $P$. falciparum, $P$. vivax, $P$. ovale) et quartes (un accès toutes les $\mathbf{7 2}$ heures : $P$. malariae, chez l'homme et $P$. inui chez le singe, par exemple). Les Plasmodium de rongeur font une schizogonie toutes les 24 heures en donnant des accès quotidiens. Une seule espèce infectant les primates, $P$. knowlesi, donne aussi ce type d'accès. D'une façon générale le paludisme se manifeste soit comme accès palustre simple, axé sur des épisodes fébriles périodiques et les phénomènes associés (cé-

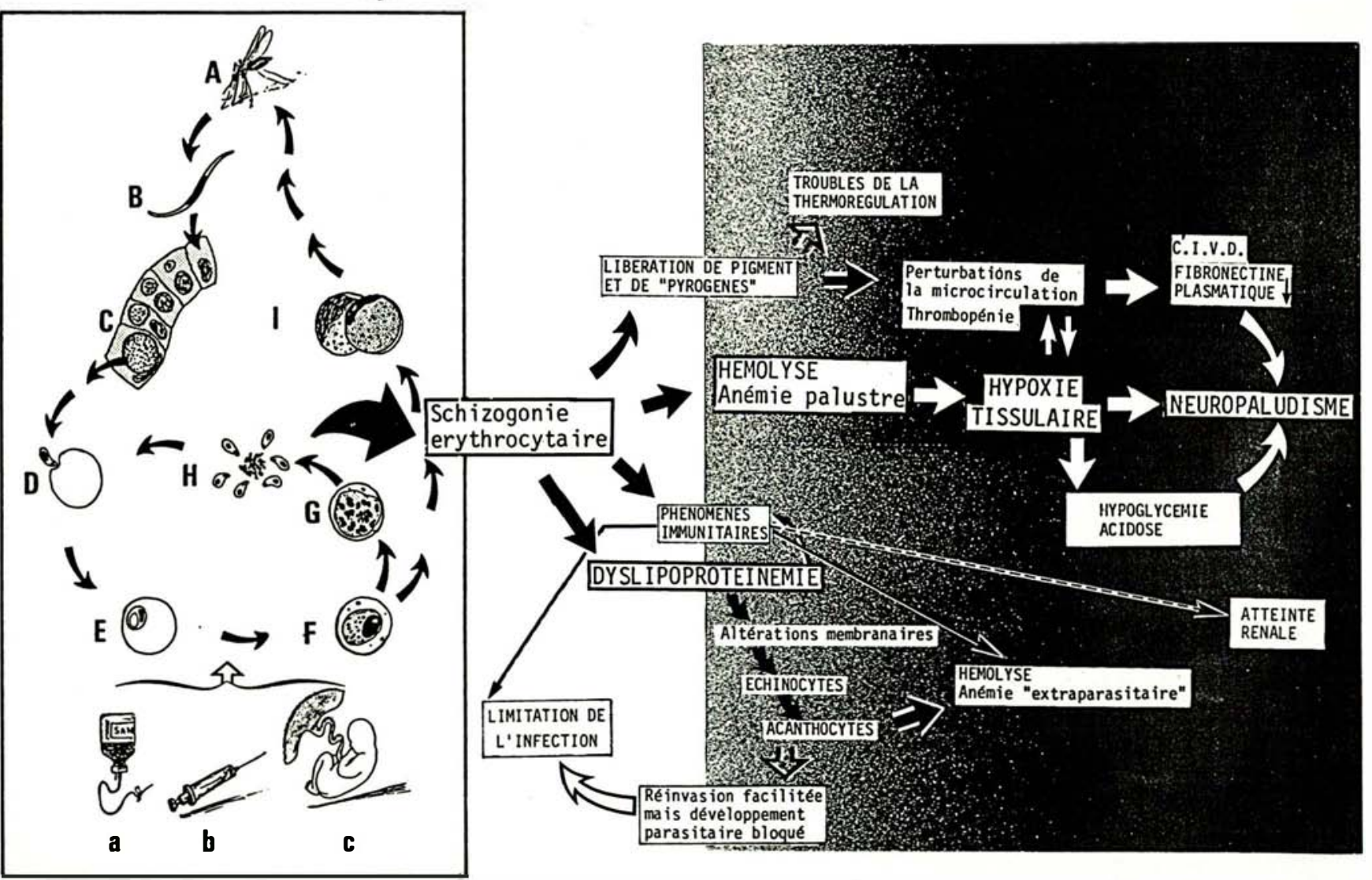

Figure 1. Cycle biologique de Plasmodium (A-I) et schéma simplifiés des principaux mécanismes physiopathologiques intervenant dans le paludisme. Cette maladie habituellement contractée par la piqûre infestante de moustiques femelles du genre Anopheles (A) peut aussi être acquise par transmission transfusionnelle (a). par la seringue (b) et par voie transplacentaire (c). Les anopheles inoculent des sporozoïtes (B) qui deviendront, dans les cellules du foie, les schizontes hépatiques (pré- ou exo-érythrocytaires) (C). Leur division asexuée donne naissance à des milliers de mérozoïtes $(D)$ capables de pénétrer dans les globules rouges où se déroule la schizogonie érythrocytaire (voir texte). E : trophozoïte jeune; $F$ : trophozoïte âgé; $G$ : schizonte; $H$ : phase finale de la division du schizonte (débris pigmentaires, rassemblés au centre, et mérozoïtes uninucléés bien individualisés): I: gamétocytes infestants pour le moustique. La schizogonie érythrocytaire et la libération de pigment et d'autres substances, déclenchent des phénomènes immunitaires et induisent des perturbations métaboliques (ex: hypog/ycémie, dyslipoprotéinémie, voir texte). Les perturbations de la microcirculation et l'hypoxie tissulaire, résultant aussi de l'anémie, sont en grande partie responsables des lésions viscérales (ex. : neuropaludisme). Les phénomènes immunitaires peuvent déterminer à long terme des troubles divers dont nous n'avons retenu dans le schéma que l'atteinte rénale (voir texte). 


\section{REFERENCES}

I. Garnham PCC. Life cycles. In: Peters W, Richards WHG, eds. Antimalarial Drugs 1. Berlin: Springer-Verlag, 1984: 3-30.

2. Landau I, Boulard Y. Life cycles and morphology. In: Killick-Kendrick R, Peters W, eds. Rodent Malaria. London: Academic Press, 1978; 53-84.

3. Trager $W$, Jensen JB. Human malaria parasites in continuous culture. Science 1976; 193: 673-5.

4. Aikawa M, Seed TM. Morphology of Plasmodia. In: Kreier JP, ed. Malaria I. New York: Academic Press, 1980; 285-344.

5. Seed TM, Kreier JP. Erythrocyte destruction mechanisms in malaria. In: Kreier JP, ed. Malaria 2. New York: Academic Press, 1980; 1-46.

6. Delvinquier B, Goumard P, Dubarry $M$, Tronchin G, Camus D. Renal deposits of lipoprotein-immunoglobulin complexes in Plasmodium chabaudi-inf ected mice. 7 Immunol 1984; 133: 2243-9.

7. Aikawa M, Suzuki M, Gutierrez Y. Pathology of malaria. In: Kreier JP, ed. Malaria 2. New York: Academic Press, r980; 47-102.

8. Boonpucknavig V, Srichaikul T, Punyagupta S. Clinical pathology. In: Peters W, Richards WHG, eds. Antimalarial Drugs I. Berlin: Springer-Verlag, 1984: 1 27-76.

9. Haque Begum S. Observations morphologiques et expérimentales sur les gamétocytes des Plasmodium de mammiferes. Thèse ( $3^{e}$ Cycle). Lille, 1984 .

10. Krishna S, Shoubridge EA, White NJ, Weatherall DJ, Radda GK. Plasmodium yoelii: Blood oxygen and brain function in the infected mouse. Exp Parasitol 1983; 56: 391-6.

I I. White NJ, Warrell DA, Chanthavanich P, et al. Severe hypoglycemia and hyperinsulinemia in falciparum malaria. $N$ Engl 7 Med 1983; 309: 6i-6.

12. Sadun EH, Williams JS, Meroney FC, Hutt G. Pathophysiology of Plasmodium berghei inf ection in mice. Exp Parasitol 1965; 17 : 277-86.

13. Maurois P, Pessah M, Briche I, Alcindor LG. Alterations of lecithin cholesterol acyltransferase activity during Plasmodium chabaudi rodent malaria. Biochimie $1985 ; 67$ : 227-39.

14. Lozano $F$, Leal $M$, Lissen $E$, Munoz J, Bautista A, Regordan C. Paludisme à P. falciparum et à $P$. malariae compliqué par un œedème pulmonaire avec coagulation intravasculaire disséminée. Presse Med 1983; $12: 3004-5$.

15. Pierach CA, Rosborough TK. Malaria, haematin, and coagulation. $\mathrm{Br}$ Med 7 1985; 290: phalées, tremblements, sudation...) soit, beaucoup moins fréquemment, comme accès pernicieux ou neuropaludisme, déterminé par $P$. falciparum, avec trouble de la conscience s'accompagnant de perturbations très importantes du milieu intérieur et de lésions du système nerveux et d'autres organes profonds. Les raisons de l'évolution vers l'un ou l'autre type d'accès demeurent obscures. La fièvre serait consécutive à la libération de "pyrogènes " lors des schizogonies. Autant chez l'homme que chez l'animal, les mécanismes de la dysrégulation thermique demeurent cependant inconnus.

L'anémie palustre peut être très importante. Elle est souvent responsable, chez $P$. falciparum et chez les espèces virulentes d'autres mammifêres, d'une très sévère hypoxie tissulaire ( figure I ). Cependant, d'autres facteurs, comme le pigment malarique, induisent de multiples et complexes changements physiopathologiques et également immunopathologiques intéressant tous les parenchymes.

\section{Les mécanismes physiopathologiques}

Nos connaissances sur la physiopathologie du paludisme proviennent en général d'études minutieuses de l'évolution de l'infection chez l'animal ou du suivi de cas humains privilégiés. La découverte à partir des années 50 d'espèces plasmodiales chez des rongeurs africains, et l'établissement de leur cycle en laboratoire [2], ont constitué des contributions d'une grande importance. La mise au point récente de techniques de culture in vitro des formes érythrocytaires [3] de Plasmodium fournit également un nouveau système qui permet de mieux cerner les rapports hôte-parasite, ceux-ci étant étroitement liés aux mécanismes physiopathologiques.

La pénétration des mérozoïtes de Plasmodium dans les hématies et leur développement induisent chez celles-ci des modifications typiques de leur taille, de leur affinité tinctoriale et de leur forme, et l'apparition de granulations. Le type d'anomalie [4] varie avec l'espèce plasmodiale. Le développement plasmodial con- duit à la production du pigment malarique (de la ferriprotoporphyrine IX associée à une fraction protéique) libéré lors de la séparation des mérozoïtes. Des phénomènes immunopathologiques peuvent découler de la réponse de l'hôte vis-àvis de l'hématozoaire (parasite du paludisme) [5] ou de la présence de substances anormales dont la synthèse a été induite chez l'hôte par le parasitisme [6]. Lors d'infections sévères, la destruction des érythrocytes parasités et non parasités, les perturbations de la microcirculation induites par l'anoxie [7], le pigment ou le simple contact des globules rouges parasités avec les endothéliums vasculaires [8] sont associées à des lésions viscérales (système nerveux, rein, rate et organes lymphatiques et hémopoiétiques, foie, appareil digestif, cœur et poumon). La nature de ces désordres et leurs conséquences histopathologiques ont été abondamment décrites chez plusieurs hôtes et ont fait l'objet de révisions récentes $[7,8]$. Cependant, le substratum physiopathologique de ces anomalies est beaucoup plus rarement abordé [7].

\section{Perturbations du milieu intérieur}

La pathogénicité des Plasmodium est liée à leur capacité à se développer dans les érythrocytes. Néanmoins, le paludisme constitue beaucoup plus qu'une érythrocytopathie et un déséquilibre de l'homéostasie du milieu intérieur s'installe fréquemment au cours du développement parasitaire.

Gaz et pH sanguins : chez $P$. yoelii nigeriensis [9], nous avons constaté que, tandis que la $\mathrm{PO}_{2}$ subit des variations faibles, la $\mathrm{PCO}_{2}$ augmente légèrement dès le début de l'infection, cette augmentation devenant significative dans le sang artériel lorsque la parasitémie dépasse $40 \%$.Le pH sanguin diminue aussi significativement. Ces changements sont associés à une nette tendance à l'augmentation de la lacticidémie. L'acidose constatée ( figure 2), respiratoire et métabolique, existe aussi chez les souris infectées par $P$. chaubaudi. Tandis que chez celles infectées par $P$. 


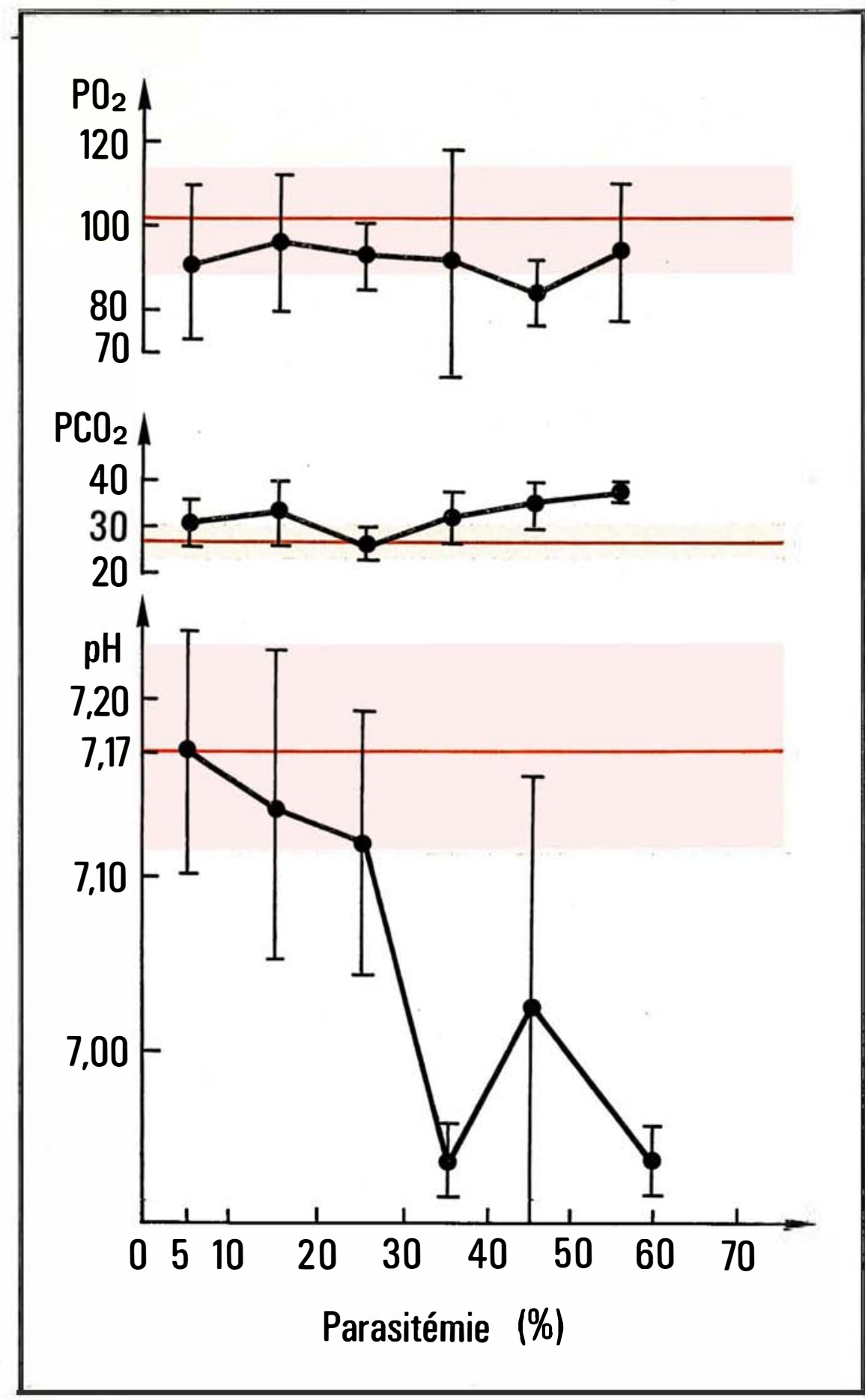

Figure 2. Evolution de la $\mathrm{PO}_{2}$ ( $\mathrm{mm} H \mathrm{H}$ ), de la $\mathrm{PCO}_{2}$ ( $\mathrm{mm}$ HG) et du pH du sang artériel au cours du paludisme mortel à Plasmodium yoelii nigeriensis chez la souris C-57 Black. Dans chaque diagramme, la droite rouge horizontale représente la valeur normale et la surface rosée représente l'écart type enregistré chez les souris saines de la même souche.

$\mathrm{m} / \mathrm{s} n^{\circ} 6$, vol. 2, juin-juillet 86 yoelii nigeriensis ou par P. yoelii yoelii [ro] l'acidose est irréversible et progresse jusqu'à la mort des animaux, chez ceux infectés par $P$. chabaudi, qui détermine une infection non fatale, une nette alcalose succède à l'acidose pendant la période de guérison.

Variations de la glycémie: beaucoup d'autres anomalies du milieu intérieur de l'hôte s'associent au développement plasmodial. Une des plus spectaculaires concerne la glycémie. Dans quelques cas de paludisme pernicieux à $P$. falciparum, une hypoglycémie inférieure à $0,4 \mathrm{~g} / \mathrm{l}$ a été rapportée [I I]. Chez ces patients, le taux de mortalité a été plus fort que dans les cas pernicieux non hypoglycémiques. L'hypoglycémie était associée à une hyperinsulinémie. Les réserves de glycogène n'étaient pas épuisées. Les troubles microcirculatoires et l'anoxie tissulaire qui en résulte semblent activer la glycolyse anaérobie en augmentant la consommation de glucose et en produisant une forte lacticidémie. Les perturbations hépatiques et l'acidose lactique pourraient bloquer les mécanismes glycogénolytiques et néoglycogéniques compensateurs de l'hypoglycémie [I I].

Ces constatations ont été approfondies grâce au paludisme expérimental. L'infection déterminée par $P$. chabaudi évolue habituellement de façon bénigne; cependant 2 à $5 \%$ des souris inoculées font une infection mortelle. Chez ces animaux, nous avons constaté une hypoglycémie associée à une forte hyperinsulinémie. Au cours de l'infection non fatale à $P$. chabaudi, la glycémie subit aussi des variations. L'hypoglycémie maximale se produit après le pic de parasitémie, lorsque le processus de guérison est déjà engagé. L'hypoglycémie est précédée et suivie d'une hypoinsulinémie mais, pendant l'hypoglycémie, l'insuline sanguine augmente en dépassant légèrement le taux normal ( figure 3 , p. suivante).

Paradoxalement, d'autres auteurs [12] ont rapporté chez l'homme une hyperglycémie survenant au cours du paroxysme fébrile. Elle pourrait être due soit à l'augmentation de la glycogénolyse associée à la stimula- 
tion du métabolisme qu'accompagne tout syndrome fébrile, soit à l'hypersécrétion d'adrénaline. Une hyperglycémie ne semble pas exister au cours de formes algides de paludisme à $P$. chabaudi. Toutefois, chez l'homme infecté par $P$. falciparum et chez la souris infectee par $P$. chabaudi, la survenue d'une hypoglycémie importante est associée à une évolution particulièrement grave et un pronostic sévère.

Protéines sériques : au cours de l'infection par $P$. chabaudi les protéines totales demeurent normales jusqu'au pic de parasitémie. Après celui-ci, leur concentration sérique s'élève [ 13$]$ redevenant normale vers le $12^{\mathrm{e}}$ jour. Ceci indiquerait que le retentissement hépatique, important à en juger par l'augmentation de TGO (transaminases glutamiqueoxaloacétiques) et TGP (transaminases glutamique-pyruviques) [13], n'entraîne pas une altération significative de la capacité de synthèse protéique de l'hépatocyte. Chez l'homme, lors de l'infection par $P$. falciparum, une hypoprotéinémie a été rapportée avec une importante réduction de l'albuminémie (jusqu'à Io g/l) [8]. Cette dysprotéinémie, responsable d'hypo-oncoticité plasmatique [14], pourrait être à l'origine des œdèmes aigus du poumon observés parfois dans le paludisme grave [14] et aurait de multiples causes [8] : réduction de la synthèse d'albumine par dysfonctionnement hépatique; augmentation du catabolisme des protéines sériques, ou "fuite " de celles-ci par augmentation de la perméabilité vasculaire.

Equilibre hydroélectrique : Des troubles du métabolisme hydrosalin ont également lieu : augmentation du volume plasmatique et hyponatrémie dans les formes cliniques bénignes; hypovolémie, déshydratation et choc hypotensif dans les formes graves avec augmentation de la perméabilité capillaire [8]. Chez les souris infectées par $P$. chabaudi le volume plasmatique augmente le jour du pic de parasitémie* et reste augmenté jusqu'au 14 jour quand les animaux sont définitivement guéris [13]. Chez l'homme, l'hyponatrémie et l'hypotension observées

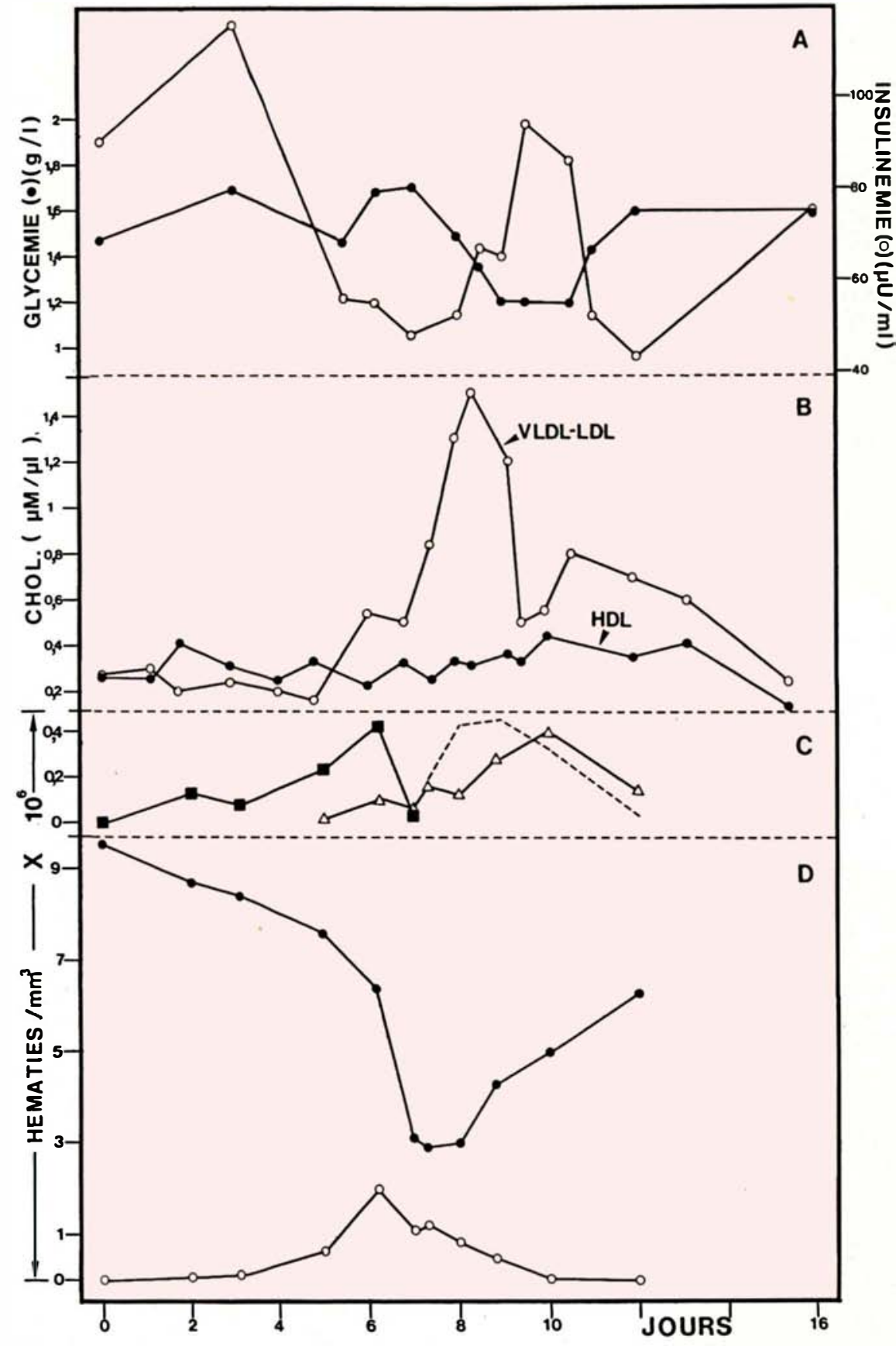

Figure 3. Paludisme bénin expérimental à Plasmodium chabaudi chez la souris Swiss. Evolution de plusieurs paramètres sanguins en fonction du temps. L'infection par inoculation intra-péritonéale de sang parasité a été réalisée le jour 0 . Les écarts-type ont été omis pour alléger la représentation. A : variations de la glycémie (cercles pleins) et de l'insulinémie (cercles vides) (selon Maurois et Portha). B : changements du cholestérol (CHOL) des VLDL-LDL (cercles vides) et des HDL (cercles pleins). Remarquer l'importante augmentation du cholestérol VLDL-LDL après le pic de parasitémie (voir $D$ ) et la chute du cholestérol HDL après le 12ème jour. $C$ :étude quantitative des anomalies érythrocytaires : échinocytes (carrés pleins) et acanthocytes (triangles vides). La courbe discontinue représente des échinocytes en cours d'acanthocytose (voir texte). D: globules rouges parasités (cercles vides) et non parasités (cercles pleins). 
lors de formes cliniques graves pourraient être en rapport avec une insuffisance cortico-surrénalienne. Ces glandes subissent en effet déplétion lipidique, oedème, thrombose, hémorragie, nécrose et infiltration cellulaire [8].

Troubles de la coagulation et autres perturbations : d'autres perturbations du milieu intérieur résultent d'atteintes viscérales spécifiques qui peuvent compliquer les formes cliniques sévères. La bilirubine peut être augmentée [13]. Protéinurie et hyperazotémie accompagnent l'insuffisance rénale aiguë souvent associée au syndrome de coagulation intravasculaire disséminée. Ce dernier semble être en rapport avec la libération de substances thromboplastiques lors de l'hémolyse intravasculaire massive, avec l'activation du complément et l'augmentation brutale de l'histamine sanguine [8]. A ces facteurs il faut ajouter le rôle du pigment malarique qui augmenterait la coagulabilité plasmatique [ $\left.{ }^{5} 5\right]$. Les troubles tissulaires seraient en grande partie dus à l'anoxie, qui est la conséquence de l'anémie, des perturbations microcirculatoires et de phénomènes cytotoxiques.

\section{La dyslipoprotéinémie au cours du paludisme}

D'après certains travaux réalisés dans notre laboratoire sur le paludisme expérimental et celui de l'homme, il semblerait que toutes les phases du métabolisme lipidique (absorption, biosynthèse intestinale, transport lymphatique et sanguin, incorporation dans les tissus, stockage et élimination) soient perturbées au cours du paludisme. Les lipides étant fortement impliqués dans le métabolisme énergétique, dans l'immunité, et dans la structure des systèmes membranaires, il est fort probable que les perturbations de leur métabolisme et de celui des lipoprotéines vectrices entraînent des effets d'importance physiopathologique primordiale.

Nature de la perturbation : les changements lipidiques induits par le paludisme sont essentiellement une augmentation des lipoprotéines

\section{* GLOSS AIRE*}

Acanthocytes : érythrocytes crénelés avec des spicules irrégulièrement disposés et renflés à leur extrémité. Cette anomalie morphologique est irréversible.

Acanthocytose : processus pathologique entraînant la formation d'acanthocytes.

Discocyte : érythrocyte biconcave, ayant une morphologie normale.

Echinocytes : érythrocytes crénelés avec des spicules régulièrement disposés. La transformation discocyte-échinocyte est réversible. Gamétocytes : cellules sexuées de Plasmodium, infestantes pour l'anophèle.

Hypnozoütes : formes latentes mononucléées intrahépatocytaires de Plasmodium, dont la réactivation serait responsable de vraies rechutes. Les hypnozoïtes se différencient à partir des sporozoïtes. Macro-réticulocytes : réticulocytes de volume augmenté. Ces cellules apparaissent lors de crises hémolytiques ou d'hémorragies aiguës.

Mérozoïtes : petites cellules uninucléées de Plasmodium résultant de la division d'un schizonte exoérythrocytaire ou érythrocytaire avant de pénétrer dans une nouvelle cellule-hôte.

Microcytes : érythrocytes de petit volume $\left(<85{\mu m^{3}}^{3}\right)$.

Normocytes : érythrocytes normaux.

Parasitémie : proportion de globules rouges parasités.

Rechutes : accès palustres survenant après disparition des premières crises et correspondant à la même infection. La reprise de la maladie peut être la conséquence soit de la réactivation d'hypnozoïtes ("vraie" rechute ou "relapse" chez les anglosaxons) soit de la persistance des parasites dans le sang (recrudescence).

Schizocytes : fragments de discocytes avec deux à quatre spicules. Schizogonie : processus de division multiple dans lequel les divisions nucléaires précèdent la division cytoplasmique : celle-ci a lieu en fin du processus qui laisse chez Plasmodium une masse cytoplasmique résiduelle, contenant le pigment malarique.

Schizonte : forme plasmodiale asexuée, après la division nucléaire.

Sphérocyte : érythrocyte sphéroidal de grande épaisseur.

Sporozoitte: forme infestante des sporozoaires. Chez Plasmodium, elle résulte de l'évolution du parasite chez l'anophèle.

Trophozoïte : forme asexuée uninuclée de Plasmodium.
$A G$ : acides gras.

CM : chylomicrons.

$H D L$ : lipoprotéines de haute densité (High Density Lipoproteins). Ig : immunoglobuline.

LDL : lipoprotéines de basse densité (Low Density Lipoproteins). $L C A T$ : lécithine-cholestérolacyl-transférase.

$L P L$ : lipoprotéine-lipase.
Plasmodium spp : espèces $d u$ genre Plasmodium.

$T A G$ : triacylglycérides.

TGO : transaminases glutamique-oxaloacétiques.

TGP : transaminases glutamique-pyruviques.

$V L D L$ : lipoprotéines de très basse densité (Very Low Density Lipoproteins). 
riches en triacylglycérides (TAG) : chylomicrons (CM) et Very Low Density Lipoproteins (VLDL). Celle-ci est associée à une augmentation des Low Density Lipoproteins (LDL) et à une chute des High Density Lipoproteins (HDL), ces deux dernières classes de lipoprotéi- nes étant riches en cholestérol. Ces modifications, nettes chez la souris Swiss infectée avec $P$. chabaudi [I6] ( figure 3), sont aussi observées chez l'homme au cours d'infestations naturelles à $P$. falciparum, $P$. malariae, $P$. vivax et $P$. ovale [17]. Chez la souris impaludée, la
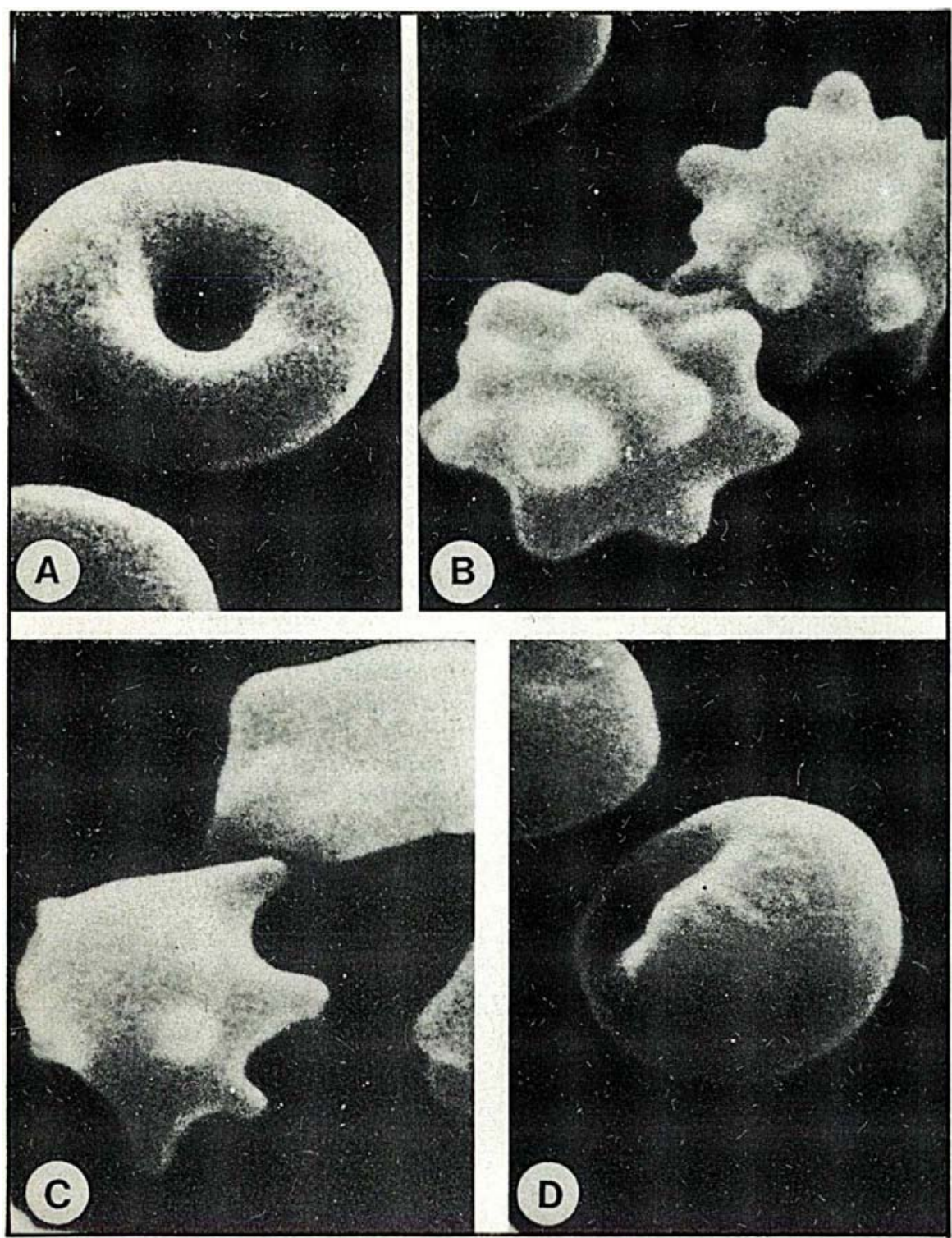

Figure 4. Modifications des hématies non parasitées au cours du paludisme à Plasmodium chabaudi chez la souris. A: normocytes; $B$ : échinocytes, nombreux 2 à 3 jours après inoculation et autour du pic de parasitémie; $C$ : acanthocytes, nombreux après ce pic; $D$ : sphérocytes. Acanthocytes et sphérocytes constituent des états anormaux irréversibles : ces cellules seront séquestrées et détruites dans la rate. dyslipoprotéinémie s'installe rapidement, est réversible et a une traduction hématologique. Ceci étend l'intérêt du phénomène au-delà de la malariologie.

Hypothèses explicatives : chez les souris infestées par $P$. chabaudi, une augmentation du taux d'acides gras (AG) libres sériques a lieu précocement lorsque la parasitémie est inférieure à I $\%$. Tout se passe comme si le parasite, incapable de synthétiser les $A G$, induisait, dès le début de l'infection, leur mobilisation à partir du tissu adipeux de l'hôte afin de satisfaire ses besoins. Ces AG en excès relatif seraient captés par le foie et transformés en TAG, car le taux hépatique de ces substances est deux fois supérieur au taux normal entre les $4^{\mathrm{e}}$ et $6^{\mathrm{e}}$ jours après l'infestation [13]. Cette vraie stéatose hépatique est transitoire. Elle serait due à un blocage de la biosynthèse des VLDL induit par le paludisme. C'est seulement après le pic de parasitémie que l'on observe un excès des VLDL sériques (figure 3) témoignant du déblocage de leur biosynthèse. L'augmentation du taux sérique de VLDL et des CM est également favorisée par l'inhibition de deux des enzymes qui participent à leur catabolisme, la lipoprotéine-lipase (LPL) [18] et la lécithine-cholestérol acyl-transférase (LCAT) [13]. Cette inhibition est très probablement due à des substances qui sont libérées lors des schizogonies.

\section{Les perturbations érythrocytaires}

La pénétration et le développement intraérythrocytaire de Plasmodium déterminent des modifications des cellules-hôtes, et constituent la cause principale de l'anémie palustre. Bien que non expliquées, les modifications des érythrocytes parasités ont été abondamment décrites [7]. Nous rappellerons l'existence de " knobs" ou excroissances, localisées sur le plasmalemme des hématies parasitées $[7,19,20]$. Ces excroissances sont responsables de l'adhésion des érythrocytes parasités aux endothéliums capillaires [7] et peuvent expliquer la séquestration des hématies parasitées dans 
les capillaires viscéraux. Le paludisme induit aussi une dysérythropoïèse, une hyperactivité du système réticulo-endothélial [8] et des modifications des érythrocytes non parasités qui conduisent à leur lyse et constituent des facteurs étiologiques importants de l'anémie. Les modifications des hématies non parasitées concernent leurs membranes et leur métabolisme oxydatif [2I] et hydroélectrolytique [22]. Ces cellules peuvent également être lysées par des mécanismes immunologiques.

Nous commenterons ici uniquement les modifications membranaires que nous avons observées récemment (résultats non publiés), les autres altérations ayant fait l'objet de revues récentes [22]. Les transformations de la morphologie des érythrocytes non parasités et parasités de souris infectées par Plasmodium chabaudi sont apparentes à l'examen microscopique. Deux jours après l'inoculation, les hématies se transforment ( figure 4), dans une proportion importante, en échinocytes*. Plus tard, la morphologie érythrocytaire redevient normale, mais lors du pic de parasitémie, des échinocytes en processus d'acanthocytose* réapparaissent et sont rapidement remplacés par des acanthocytes* associés à des microcytes*, schizocytes* et sphérocytes*. Une augmentation du rapport membranaire cholestérol/phospholipides est constatée dans les populations acanthocytaires. Enfin, au cours de la guérison, ces érythrocytes anormaux sont graduellement ręmplacés par des macro-réticulocytes*.

A notre connaissance, c'est la première fois que des transformations morphologiques bien caractérisées des hématies non parasitées, associées à des perturbations de leur composition membranaire, sont rapportées au cours du paludisme. Ces anomalies apparaissent liées aux perturbations des lipoprotéines sériques qui existent aussi chez l'homme et chez le singe impaludés [17], où cependant nous n'avons pas encore pu les retrouver. Les modifications membranaires lipidiques que nous avons évoquées pourraient influencer le cours de la parasitose. Lors de l'infection à $P$. chabaudi, les échinocytes et les $m / s n^{\circ} 6$, vol. 2, juin-juillet 86 acanthocytes sont, par rapport aux discocytcs*, plus souvent envahis par Plasmodium. Cependant les parasites n'atteignent jamais l'état de schizonte* mature dans les cellules anormales. Le processus d'acanthocytose chez $P$. chabaudi pourrait en partie expliquer l'évolution bénigne de l'infection : des parasites en grand nombre seraient piégés dans les globules rouges anormaux, ceux-ci étant ensuite rapidement séquestrés par la rate.

\section{Aspects immunopathologiques}

Les modifications du fonctionnement immunitaire au cours du paludisme sont variées et complexes. Leurs répercussions pathologiques s'expriment à court ou à long terme. A court terme, les manifesta-

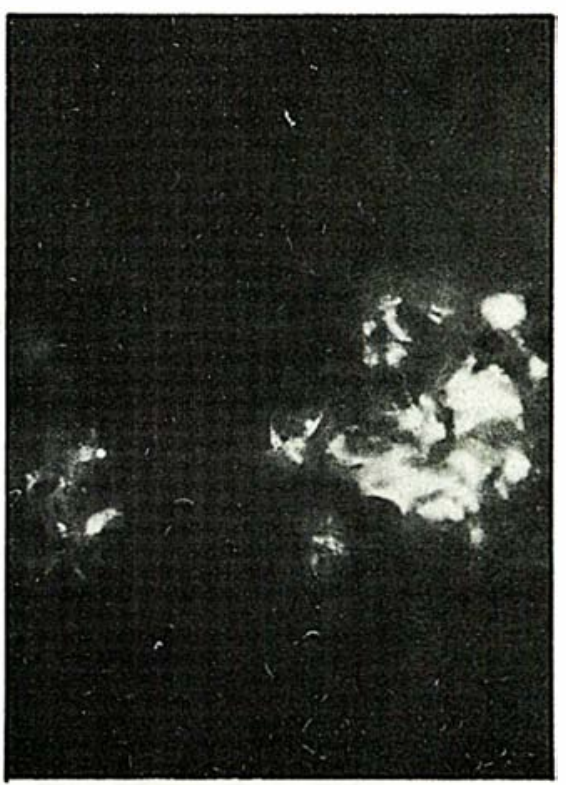

Figure 5. Paludisme bénin à Plasmodium chabaudi chez la souris Swiss. Dépôt de LDL dans le glomérule rénal 29 jours après l'inoculation intra-péritonéale de sang parasité (voir texte). La parasitémie était nulle depuis 12 jours environ. Technique d'immunofluorescence indirecte avec un anticorps anti-LDL et une anti-imriunoglobuline conjuguée à la fluorescéine $\times 660$. Cliché du Profes seur D. Camus. tions immunopathologiques les plus importantes se traduisent par une possible participation d'anticorps anti-érythrocytaires à l'hémolyse ou par l'apparition de complexes immuns pouvant retentir sur le fonctionnement rénal. Les sérums de sujets souffrant de paludisme à $P$. falciparum, $P$. vivax ou $P$. ovale contiennent souvent de hauts titres d'anticorps dirigés contre des structures des membranes érythrocytaires dont certaines ont été identifiées à la sous-unité $\alpha$ de la spectrine ou à l'actine [23]. La protéinurie contemporaine des accès palustres est d'une nature différente de celle observée lors des syndromes néphrotiques post-palustres : souvent associée à des signes de glomérulonéphrite, elle régresse rapidement sous traitement antimalarique. Des complexes immuns ont été retrouvés dans les glomérules rénaux de certains patients infectés par $P$. falciparum [24]. On peut rapprocher cette observation de celles faites lors du paludisme expérimental de la souris à $P$. chabaudi, où des complexes immuns d'immunoglobulines et de lipoprotéines anormales se déposent dans les glomérules rénaux [6] ( figure 5).

A plus long terme, le paludisme induit de profondes modifications de la réactivité immunologique des malades, comme en atteste l'existence d'une hypergammaglobulinémie, de phénomènes d'autoimmunité ou d'immuno-dépression [25]. La réponse humorale préexistante vis-à-vis de nombreux antigènes $T$-dépendants ou $T$-indépendants est déprimée au cours et au décours de l'infection palustre. De même, les réponses à médiation cellulaire peuvent être modifiées ainsi que la réponse des lymphocytes aux mitogènes et alloantigènes. A l'heure actuelle, aucun mécanisme ne peut à lui seul expliquer parfaitement les eff ets suppresseurs du paludisme sur la réponse immune de l'hôte. Nous signalerons la fréquence des anticorps lymphocytotoxiques chez les patients infectés par $P$. falciparum ou $P$. vivax, anticorps conduisant à une déplétion en cellules $T$ circulantes [26]. Nous avons pu nous-mêmes constater, au cours du paludisme expérimental des rongeurs (travaux non publiés), 


\section{REFERENCES}

16. Maurois $P$, Charet $P$, Nouvelot $A$, Fruchart JC, Vernes A, Biguet J. Kinetic study of serum lipoproteins, total cholesterol and triacylglycerides in various models of experimental rodent malaria. Ann Trop Med Parasitol 1980; 74: 17-28.

17. Vernes A, Dei-Cas E, Dutoit E, Maurois P, Gentilini JL, Biguet J. Modifications du lipoprotéinogramme au cours du paludisme humain. Pathol Biol 1980; 28: 457-60.

18. Al Rifai W, Maurois P, Fruchart J-CH, Charet $P$. Adipose tissue lipoprotein lipase activity in Plasmodium chabaudi and $P$. vinckei rodent malaria. Biochimie 1982; 64: 127-32.

19. Langreth SG, Reese RT, Motyl MR, Trager W. Plasmodium falciparum: Loss of knobs on the inf ected erythrocyte surf ace af ter long-term cultivation. Exp Parasitol 1971; 48: $213-9$.

20. Gruemberg J, Albrid DR, Sherman W. Scanning electron microscope-analysis of the protrusions (Knobs) present on the surface of Plasmodium falciparum inf ected erythrocytes. $7 \mathrm{Cell} \mathrm{Biol}$ 1983; 97: 795-802.

21. Dupouy-Camet J, Verdy E, Combrisson A, Lapierre J. Un argument supplémentaire en faveur de l'effet protecteur du déficit en glucose6-phosphate deshydrogénase à l'égard du paludisme? Presse Med 1984; 13 : 167.

22. Weatherall DJ, Abdalla S, Pippard MJ. The anaemia of Plasmodium falciparum malaria. In: Ciba Foundation symposium 94. Malaria and the Red Cell. London: Pitman, 1983: 74-97.

23. Berzins K, Wahlgren M, Perlmann P. Studies on the specificity of anti-erythrocyte antibodies in the serum of patients with malaria. Clin Exp Immunol 1983; 54: 313-8.

24. Allison AC, Houba V. Immunopathology due to complexes of antigen and antibody (type III reactions). In: Cohen S, Sadun E, eds. Immuno$\log y$ of Parasitic Infections. Oxford: Blackwell Scientific Publications, 1976: 436-47.

25. Weidanz WP. Malaria and alterations in immune reactivity. $\mathrm{Br}$ Med Bull 1982; 38: 167-72.

26. Wells RA, Pavanand K, Zolyomi S, Permpanich B, MacDermott RP. Anti-lymphocytotoxic antibodies in sera of Thai adults infected with Plasmodium falciparum or Plasmodium vivax. Clin Exp Immunol 1980; 39: 663-7.

27. Soulier JP, Prou O, Dubarry M, Luilier M, Canavaggio M, Lee $\mathrm{H}$. Etude immunologique du paludisme à l'aide d'anticorps monoclonaux. A propos d'un cas d'accès pernicieux à Plasmodium falciparum. Sem Hop Paris 1984; 60: 1659-63.

28. Ouaissi MA, Dutoit E, Vernes A, Guerret Stocker S, Capron A. In vitro inhibition of Plasmodium falciparum merozoite invasion by human plasma fibronectin. Parasitol 1985; 90: 227-36. que certaines sous-classes de lipoprotéines anormales (VLDL-Ln) apparaissant en cours d'infection, possédaient un rôle suppresseur important vis-à-vis de différentes fonctions immunologiques, comme la réponse humorale préexistante vis-à-vis de différents antigènes ou la réponse lymphocytaire aux mitogènes. Cette activité immuno-suppressive a pu être liée à une avidité vingt fois supérieure, par rapport aux lipoprotéines des animaux sains, de ces lipoprotéines "palustres" pour les récepteurs lymphocytaires. Le paludisme est bien connu pour augmenter la susceptibilité des individus qui en sont atteints vis-à-vis d'une grande variété d'autres maladies infectieuses. L'immunosuppression déclenchée par l'infection palustre est très probablement la cause de ces surinfections, comme le suggèrent nombre de résultats expérimentaux [25], mais il est difficile de tirer des conclusions définitives de ces travaux, effectués pour la plupart sur des modèles de rongeurs ou de primates inférieurs, qui ne peuvent être entièrement extrapolés au paludisme humain. L'association entre le paludisme et le lymphome de Burkitt ou les infections à virus d'Epstein-Barr représente un bon exemple d'un domaine de recherches qui a largement stimulé l'étude des phénomènes d'immunosuppression induits par le paludisme. Le syndrome néphrotique est une des complications classiques à long terme du paludisme. Relativement fréquent lors des infections chroniques dues à $P$. malariae, il est en revanche rarement observé avec $P$. falciparum. Antigènes et anticorps spécifiques associés au complément ont été détectés par immunofluorescence dans les glomérules rénaux [24].

Enfin, bien que n'appartenant pas au domaine de l'immunopathologie, nous mentionnerons ici l'effondrement des taux de fibronectine plasmatique récemment décrit chez les sujets atteints de paludisme sévère à $P$. falciparum [27]. Ce phénomène est sans doute en rapport avec l'existence d'un récepteur pour la fibronectine plasmatique humaine à la surface des mérozoïtes de $P$. falciparum $[28]$

\section{Summary}

The clinical signs of malaria correspond to the relatively precise chronobiology of the erythrocytic schizogony of Plasmodium. Although anemia is chiefly due to the multiplication of the parasite within the red blood cells, other factors are involved. Plasmodial development leads to the production of malarial pigment. Its release, with that of other substances, is associated with visceral lesions. Perturbations of homeostasis such as acidosis with hyperlacticidemia are associated with serious clinical manifestations. Hypoglycemia and hyperinsulinemia are associated with pernicious forms, whose prognosis is of ten fatal. Plasmodium spp. induces modifications in serum lipoproteins in the mouse, the primates and man. In $P$. chabaudi infected mice these modifications are associated with the occurence of echinocytes and acanthocytes. Malaria causes marked perturbations in the over-all immunological reactivity of the host. Some humoral responses can be responsible for immunopathological disorders such as the nephrotic syndrome or glomerulonephritis. Abnormal lipoproteins participate in immunosuppression and deposits of immune complexes in the glomeruli. Anti-lymphocytic antibodies lead to $\mathrm{T}$-cell depletion and could play a role in the immunosuppression. Antibodies related to spectrin or actin of red blood cells could also explain partly the anemia not directly related to the growth of the parasites within the red blood cells.

\section{TIRES A PART}

A. Vernes : Inserm U 42, 369 rue J. Guesde, 59650 Villeneuve-d'Ascq. 